\title{
Ventilación mecánica en pacientes con patologías agudas del Sistema Nervioso Central: sobrevida y pronóstico funcional
}

\section{Enrique Norero $\mathrm{M}^{\mathrm{a}}$, Pablo Altschwager $\mathrm{K}^{\mathrm{a}}$, Carlos Romero $\mathrm{P}^{1}$, Patricio Mellado $\mathrm{T}^{2}$, G lenn Hernández $\mathrm{P}^{1,3}$, Luis Castillo $\mathrm{F}^{\mathbf{1}, 3}$, G uillermo Bugedo $\mathrm{T}^{1,3}$.

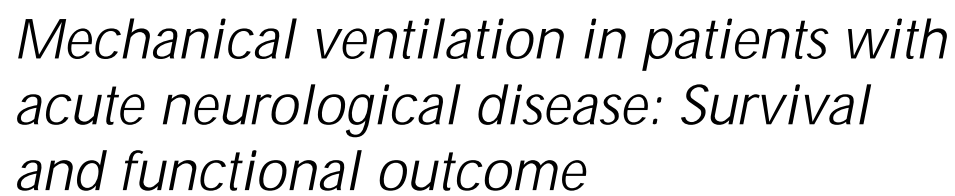

Background: The need of mechanical ventilation among patients with acute neurological diseases is considered a poor prognostic sign. Aim: To determine the mortality and functional recovery of neurological patients requiring mechanical ventilation. Patients and methods: Prospective study of 77 patients ( 42 men, age $54 \pm 19$ years, with $11 \pm 4$ points of Glasgow coma scale (GCS), $61 \%$ with cerebrovascular disease), that were admitted to the intensive care unit with neurological disease and that required mechanical ventilation. Functional recovery was assessed at 18 months with Glasgow outcome scale (GOS) and Barthel index. Results: Thirty percent of patients died during follow up. Among surviving patients, $47 \%$ had a good recovery or moderate disability, and $74 \%$ had a Barthel index equal to or over 70 . Arterial hypertension, age over 70 and mechanical ventilation longer than 6 days were associated with bad functional prognosis. Conclusions: Neurological patients requiring mechanical ventilation had a lower mortality than previously reported, and half of the survivors have an independent life. This study supports intensive care management in this group of patients (Rev Méd Chile 2004; 132: 11-8).

(Key Words: Intensive care; Neurologic manifestations; Ventilators, mechanical)

\begin{abstract}
Recibido el 11 de junio, 2003. Aceptado en versión corregida el 5 de noviembre, 2003.
Programa de Medicina Intensiva ${ }^{1}$. Departamentos de Neurología ${ }^{2}$ y de Anestesiología ${ }^{3}$, Facultad de Medicina, Hospital Clínico, Pontificia Universidad Católica de Chile. Santiago, Chile.

anternos de $7^{0}$ año de la Escuela de Medicina.
\end{abstract}

L a necesidad de ventilación mecánica (VM) en el curso de una enfermedad neurológica aguda denota un cuadro grave con elevada morbimortalidad y limitada recuperación funcional ${ }^{1,2}$. Un alto porcentaje de los pacientes presentan compromiso

Correspondencia a: Dr. Guillermo Bugedo. Marcoleta 367, Santiago. Casilla 114-D. E mail: bugedo@med.puc.cl variable del centro respiratorio, con depresión ventilatoria e hipoventilación, pudiendo además desarrollar hipoxemia debido a múltiples complicaciones respiratorias, como atelectasias, retención de secreciones, infecciones, síndrome de distrés respiratorio agudo (SDRA) y tromboembolismo pulmonar, empeorando la condición neurológica, y condicionar un círculo vicioso de difícil manejo ${ }^{3}$. 
En el curso de los últimos cinco años, hemos implementado un protocolo prospectivo de manejo de los pacientes neurológicos graves que implica ingresar a una Unidad de Cuidados Intensivos (UCI) y el soporte agresivo de la función ventilatoria entre otras medidas.

Nuestro objetivo fue determinar la evolución a corto (durante la hospitalización) y largo plazo (18 meses de seguimiento) de los pacientes que ingresaron a nuestra unidad por un cuadro neurológico agudo y que requirieron de $\mathrm{VM}$, en términos de mortalidad y de recuperación funcional.

\section{PACIENTES Y MÉTODOS}

Llevamos a cabo un estudio prospectivo observacional. Incluimos a todos los pacientes que ingresaron entre junio de 1999 hasta abril de 2001 a la Unidad de Cuidados Intensivos Quirúrgicos del Hospital Clínico de la Universidad Católica por patología neurológica y que requirieron de VM por más de $24 \mathrm{~h}$.

Protocolo de manejo ventilatorio. Básicamente, los pacientes son intubados y conectados a VM por motivos neurológicos si el puntaje en la escala de coma de Glasgow (GCS) es menor a 84 . Pacientes con GCS entre 9 y 14 son intubados sólo si existe hipoxemia o evidencias de un trabajo respiratorio aumentado. Los pacientes son ventilados inicialmente con volumen corriente de $8 \mathrm{ml} / \mathrm{kg}$, ajustándolo para mantener presiones meseta bajo 25 $\mathrm{CmH}_{2} \mathrm{O}$. Todos son ventilados con presión positiva al final de espiración (PEEP) de al menos 5 $\mathrm{cmH}_{2} \mathrm{O}$, y la fracción inspirada de oxígeno $\left(\mathrm{FiO}_{2}\right)$ ajustada para mantener la saturación de $\mathrm{O}_{2}$ sobre 90\%. La modalidad preferida es la presión de soporte inspiratoria (PS) con ventilación mandatoria intermitente (IMV), la cual se disminuye progresivamente. Los pacientes son extubados al obedecer órdenes y tolerar bajos niveles de PS y PEEP. Sólo aquellos pacientes que requieren traqueostomía (TQT) son desconectados a tubo T. Se consideró el «destete» cuando se toleró 1 día de ventilación espontánea sin apoyo.

Recolección de información. Las variables fueron ingresadas prospectivamente en una base de datos de pacientes sometidos a VM de nuestra unidad, registrándose información demográfica, tipo de patología neurológica, antecedentes de diabetes mellitus (DM), hipertensión arterial crónica (HTA), y accidente vascular encefálico previo (AVE). Al ingreso se evaluó el GCS, el examen físico neurológico, puntajes APACHE II ${ }^{5}$ y SOFA 6 del primer y tercer día en la UCI.

Consignamos la indicación de VM (neurológica versus respiratoria) y el momento de la conexión a ésta. Registramos en forma diaria los siguientes parámetros: presión media de vía aérea (PVA), PEEP, la fracción inspirada de oxígeno $\left(\mathrm{FiO}_{2}\right)$ y modalidad del ventilador. Se calculó la distensibilidad tóraco-pulmonar (DistTP $\left.=\mathrm{Vt} / \mathrm{P}_{\text {mes }}-\mathrm{PEEP}\right)$, con el volumen corriente (Vt) y la presión meseta de la vía aérea $\left(\mathrm{P}_{\mathrm{mes}}\right)$ medida después de una pausa inspiratoria de 2 a $3 \mathrm{~s}$. También se registró en forma diaria la $\mathrm{PaO}_{2}$, $\mathrm{PaCO}_{2}$ y $\mathrm{pH}$ en sangre arterial, con lo que calculamos la relación $\mathrm{PaO}_{2}: \mathrm{FiO}_{2}\left(\mathrm{~Pa} / \mathrm{FiO}_{2}\right)$ y el índice de oxigenación ${ }^{7}$.

Se consideró el término de la VM, cuando el paciente fue extubado exitosamente o respiraba espontáneamente por una traqueostomía. Se registró la incidencia de neumonía y de SDRA, así como el tiempo de estadía en la UCI, la duración de la VM y la mortalidad intrahospitalaria.

Seguimiento. Realizamos seguimiento telefónico por 2 de los autores con el fin de establecer la mortalidad a largo plazo y el grado de recuperación funcional. Para este propósito empleamos la escala de resultado de Glasgow (GOS, de su sigla en inglés Glasgow outcome scale) y el índice de Barthel (Tabla 1) ${ }^{8,9}$. Aportaron información los familiares o personas que viven con el paciente, y en el caso de los pacientes en buenas condiciones, ellos mismos.

Estadística. Realizamos análisis univariado y multivariado para identificar predictores de mortalidad y de mal pronóstico funcional, con índice de Barthel bajo 90 y 70. Los parámetros ventilatorios se compararon mediante test de t-Student. Consideramos estadísticamente significativo un valor $\mathrm{p}<0,05$. Para el análisis de sobrevida se realizó curva de Kaplan-Meier. 


\section{RESULTADOS}

Durante los 23 meses de estudio ingresaron 77 pacientes (42 hombres, 35 mujeres, $54 \pm 19$ años) con patología neurológica aguda que requirieron VM durante más de $24 \mathrm{~h}$. El GCS de ingreso fue de $11 \pm 4$ y APACHE II de $14 \pm 7$.

Las patologías neurológicas más frecuentes fueron: AVE hemorrágico (25\%), hemorragia subaracnoidea (HSA) aneurismática (21\%), AVE isquémico (16\%), traumatismo encéfalo craneano (TEC) (10\%), neurocirugía electiva debido a tumor (8\%) y otros (21\%). El grupo con patología cerebrovascular representa el $61 \%(n=47)$ de nuestra serie (Tabla 2).

La intubación se realizó durante las primeras $48 \mathrm{~h}$ en el $60 \%$ de los pacientes, y debido a indicación neurológica en $71 \%$. Los pacientes estuvieron conectados a VM durante un promedio de 5 días (rango: 1-23), con una estadía promedio en UCI de 11 días (rango: 2-39).

Encontramos complicaciones respiratorias en 15 (19\%) pacientes: 13 (17\%) neumonías nosocomiales, de las cuales 3 evolucionaron a un SDRA. La falla de «destete» se presentó en 7 (9\%) pacientes. En 9 (12\%) pacientes se decidió realizar una TQT; este grupo estuvo conectado a VM durante un promedio de 11 días (rango: 3-23), significativamente más tiempo que los pacientes sin traqueostomía ( $p<0,0001$ ).

La mortalidad intrahospitalaria alcanzó el 23\% $(n=18), y$ durante el período de seguimiento ésta aumentó a 30\% (n=23) (Figura 1). De los 18 pacientes que fallecieron tempranamente, la causa de muerte fue patología neurológica en 17 de ellos, y sólo un paciente falleció debido a una sepsis de origen pulmonar. De los 5 pacientes que fallecieron durante el seguimiento, un caso se debió a un nuevo AVE, un segundo paciente que fue dado de alta en estado vegetativo falleció debido a una neumonía, y un tercer paciente portador de una insuficiencia renal crónica falleció 2 semanas después del alta con un manejo conservador debido a sus secuelas neurológicas. En los 2 pacientes restantes no se pudo establecer la causa de muerte.

Realizamos seguimiento telefónico a 48 de los 59 sobrevivientes (81\%), en promedio 18 meses después del alta. De acuerdo a la GOS, entre los sobrevivientes, encontramos un paciente en estado vegetativo persistente, 22 (51\%) severamente discapacitados (pacientes que requieren apoyo diario de otras personas por discapacidad física, mental o ambas), y 20 (47\%) tuvieron discapaci-

\section{Tabla 1. Indice de Barthel. Puntaje máximo es de 100.}

\begin{tabular}{|lr|}
\hline Alimentación & \\
Incapaz & 0 \\
Necesita ayuda o requiere dieta modificada & 5 \\
Independiente & 10 \\
Bañarse & \\
Dependiente & 0 \\
Independiente & 5 \\
Cuidados personales & \\
Necesita ayuda & 0 \\
Independiente & 5 \\
Vestirse & \\
Dependiente & 0 \\
Necesita ayuda, pero puede hacer la mitad solo & 5 \\
Independiente & 10 \\
Continencia digestiva & \\
Incontinencia completa & 0 \\
Incontinencia ocasional & 5 \\
Continente & 10 \\
Continencia urinaria & \\
Incontinencia completa & 0 \\
Incontinencia ocasional & 5 \\
Continente & 10 \\
Uso del baño & \\
Dependiente & \\
Necesita algo de ayuda & \\
Independiente & 10 \\
Movimientos entre silla de ruedas y cama & \\
Incapaz, falta de equilibrio para sentarse & \\
Ayuda mayor (1 ó 2 personas), puede sentarse & 5 \\
Ayuda menor (verbal o física) & 10 \\
Independiente & \\
Movilidad en superficies planas & 15 \\
Inmóvil o se moviliza menos de 50 yardas & \\
Puede ocupar silla de ruedas & 0 \\
Camina con ayuda de 1 persona, sobre 50 yardas & 10 \\
Independiente & 15 \\
Movilidad en escaleras & \\
Incapaz & \\
Necesita ayuda \\
Independiente \\
\hline
\end{tabular}


dad moderada o buena recuperación (Tabla 3). El puntaje según el índice de Barthel para los sobrevivientes fue de $76 \pm 35$, encontrando $74 \%$ con un puntaje $\geq 70$ (Tabla 3 ).
En el análisis multivariado para la mortalidad, solo el puntaje de APACHE II fue significativo, en el análisis univariado el GCS al ingreso y la indicación neurológica de VM representaron tam-

Tabla 2. Patologías, mortalidad en la UT I y luego de los 18 meses de seguimiento. El grupo de pacientes con enfermedad cerebrovascular está subdividido por tipo de AVE.

\begin{tabular}{|c|c|c|c|c|c|c|}
\hline \multirow[t]{2}{*}{ Patología } & \multirow[t]{2}{*}{$\mathrm{n}$} & \multirow[t]{2}{*}{$\%$} & \multicolumn{2}{|c|}{ Mortalidad UTI } & \multicolumn{2}{|c|}{ Mortalidad 18 meses } \\
\hline & & & $\mathrm{n}$ & $\%$ & $\mathrm{n}$ & $\%$ \\
\hline Enfermedad cerebrovascular & 47 & 61 & 15 & 32 & 18 & 38 \\
\hline AVE hemorrágico & 19 & 25 & 4 & 21 & 6 & 32 \\
\hline Ganglios basales & 4 & 5 & 0 & 0 & 1 & 25 \\
\hline Infratentorial & 3 & 4 & 1 & 33 & 1 & 33 \\
\hline Lobar & 12 & 16 & 3 & 25 & 4 & 33 \\
\hline AVE Isquémico & 12 & 16 & 5 & 42 & 6 & 50 \\
\hline Supratentorial & 4 & 5 & 1 & 25 & 1 & 25 \\
\hline Infratentorial & 8 & 10 & 4 & 50 & 5 & 63 \\
\hline HSA & 16 & 21 & 6 & 38 & 6 & 38 \\
\hline TEC & 8 & 10 & 1 & 13 & 2 & 25 \\
\hline Neurocirugía por tumor & 6 & 8 & 1 & 17 & 1 & 17 \\
\hline Otros & 16 & 21 & 1 & 6 & 2 & 13 \\
\hline Total & 77 & 100 & 18 & 23 & 23 & 30 \\
\hline
\end{tabular}

TEC: Traumatismo encefalocraneano, HSA: hemorragia subaracnoidea.



FIgURA 1. Curva de sobrevida global de Kaplan-Meier, para los 77 pacientes en VM. 
Tabla 3. Resultados funcionales según escala de resultado de G lasgow e índice de Barthel.

\begin{tabular}{|lrr|}
\hline & $\mathrm{n}$ & $\%$ \\
\hline Escala de resultado de Glasgow & & \\
Buena recuperación & 12 & 25 \\
Moderadamente discapacitado & 8 & 17 \\
Severamente discapacitado & 22 & 46 \\
Estado vegetativo persistente & 1 & 2 \\
Muerte & 5 & 10 \\
Total & 48 & 100 \\
Indice de Barthel & & \\
100-90 & 28 & 65 \\
$85-70$ & 4 & 9 \\
$65-40$ & 4 & 9 \\
$0-35$ & 7 & 16 \\
Total & 43 & 100 \\
\hline
\end{tabular}

bién predictores de mortalidad (Tabla 4). Existe diferencia estadísticamente significativa para los parámetros ventilatorios; $\mathrm{PaO}_{2}, \mathrm{pH}$ arterial y dis- tensibilidad en el grupo de pacientes que fallece (Tabla 5).

Tabla 4. Regresión logística y comparación para mortalidad, luego de 18 meses de seguimiento. Sólo se muestran las variables significativas.

\begin{tabular}{|lcccc|}
\hline Variable & OR & CI & Muertos & Vivos \\
\hline Multivariado & & & & \\
$\quad$ APACHE II & 9,66 & $2,75-34,02$ & $17,1 \pm 7,4$ & $12,0 \pm 6,1$ \\
Univariado & & & & \\
$\quad$ GCS al ingreso & 0,85 & $0,73-0,96$ & $9,7 \pm 4,4$ & $12,2 \pm 3,2$ \\
$\quad$ Indicación neurológica de VM & 6,00 & $1,23-29,38$ & $90 \%$ & $62 \%$ \\
\hline
\end{tabular}

GCS: escala de coma de Glasgow.

Tabla 5. Comparación de parámetros ventilatorios promedio durante el período de VM entre pacientes vivos y muertos luego de 18 meses de seguimiento.

\begin{tabular}{|lccc|}
\hline Parámetros ventilatorios & Muertos & Vivos & $\mathrm{p}$ \\
\hline PVA $\left(\mathrm{cm} \mathrm{H}_{2} \mathrm{O}\right)$ & $8,6 \pm 1,5$ & $9,4 \pm 1,7$ & 0,0613 \\
$\mathrm{PEEP}\left(\mathrm{cm} \mathrm{H}_{2} \mathrm{O}\right)$ & $5,0 \pm 0,9$ & $5,5 \pm 1,3$ & 0,1068 \\
$\mathrm{FIO}_{2}(\%)$ & $41 \pm 9$ & $41 \pm 6$ & 0,8419 \\
$\mathrm{PaO}_{2}(\mathrm{mmHg})$ & $149 \pm 66$ & $126 \pm 27$ & $0,0465^{*}$ \\
$\mathrm{PaCO}(\mathrm{mmHg})$ & $33 \pm 4$ & $32 \pm 5$ & 0,4634 \\
$\mathrm{Ph} \mathrm{arterial}$ & $7,41 \pm 0,04$ & $7,45 \pm 0,04$ & $0,014^{*}$ \\
$\mathrm{Dist}\left(\mathrm{ml} / \mathrm{cm} \mathrm{H}_{2} \mathrm{O}\right)$ & $62,1 \pm 10,2$ & $55,7 \pm 9,4$ & $0,0467^{*}$ \\
$\mathrm{PAFI}$ & $343 \pm 66$ & $320 \pm 79$ & 0,2398 \\
$\mathrm{Iox}$ & $2,9 \pm 1,1$ & $3,2 \pm 1,3$ & 0,3255 \\
\hline
\end{tabular}

PVA: presión media de vía aérea. PEEP: presión positiva al final de espiración. Dist: distensibilidad estática toraco-pulmonar. PAFI: relación $\mathrm{PaO}_{2} / \mathrm{FIO}_{2}$. Iox: índice de oxigenación. *p $<0,05$. 
Encontramos que la edad sobre 70 años y el antecedente de HTA representaron variables que predicen en forma independiente un resultado en el índice de Barthel $<90$, mientras que la conexión a VM por sobre 6 días predice independientemente un puntaje de Barthel <70. En el análisis univariado también predicen un peor pronóstico funcional la falla del destete, el desarrollo de neumonía, y una mayor estadía en la UCI (Tabla 6).

Enfermedad cerebrovascular. El grupo de pacientes con patología cerebrovascular representa al $61 \%(n=47)$ de los pacientes (22 hombres, 25 mujeres $57 \pm 17$ años). El GCS al ingreso fue de $11 \pm 4$ puntos y el APACHE II $14 \pm 7$ puntos. Los AVE más frecuentes fueron los hemorrágicos lobares $(n=12)$ y los infartos en el territorio vertebrobasilar $(\mathrm{n}=8)$ (Tabla 2).

La estadía en la UCI y en VM fue de $10 \pm 6$ y $5 \pm 4$ días, respectivamente. En el $66 \%$ de los pacientes la VM se inició dentro de las primeras $48 \mathrm{~h}$ del ingreso al hospital, siendo el $75 \%$ ventilados por compromiso neurológico, $13 \%$ después de un procedimiento y $8 \%$ debido a patología respiratoria. Los procedimientos fueron 4 casos de HSA en que se excluyó el aneurisma por cirugía o por terapia endovascular, la instalación de un drenaje ventricular externo en un AVE isquémico del territorio vertebrobasilar complicado con hidrocefalia y el drenaje quirúrgico de un hematoma lobar.

La mortalidad para el grupo de patología cerebrovascular fue de $32 \%(\mathrm{n}=15)$ y aumentó a $38 \%(n=18)$ durante el seguimiento.

En los sobrevivientes encontramos 14 (54\%) pacientes con buena recuperación o moderada discapacidad y 12 (46\%) con discapacidad severa.

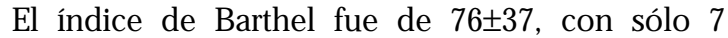
(27\%) pacientes con puntaje menor a 70.

\section{DisCUSIÓN}

Hemos mostrado que el manejo activo en UCI de pacientes con enfermedad neurológica grave que requieren apoyo con ventilación mecánica, tienen una sobrevida y recuperación funcional aceptable (moderada discapacidad o buena recuperación).

Numerosos trabajos han mostrado una morbimortalidad muy elevada en pacientes neurológicos críticos $^{10,11}$. La mortalidad global en UCI en nuestro estudio fue de $23 \%$, aumentando a $30 \%$ durante el seguimiento. En el grupo de enfermedad cerebrovascular, ésta alcanzó 32 y 38\% respectivamente. Estas cifras se comparan favorablemente con los

Tabla 6. Regresión logística y comparación de los pacientes con índice de Barthel menor de 90 y 70. Sólo se muestran las variables significativas.

\begin{tabular}{|lcccc|}
\hline Variable & OR & CI & Barthel $<90$ & Barthel $\geq 90$ \\
\hline Multivariado & & & & \\
HTA & 5,49 & $1,23-24,68$ & $75 \%$ & $28 \%$ \\
Edad $\geq 70$ & 5,43 & $1,18-24,88$ & $55 \%$ & $25 \%$ \\
& & & & \\
\hline Variable & OR & CI & Barthel $<70$ & Barthel $\geq 70$ \\
\hline Multivariado & & & & \\
$\quad$ Sobre 6 días en VM & 5,20 & $1,18-22,89$ & $55 \%$ & $19 \%$ \\
Univariado & & & & \\
$\quad$ Falla destete & 11,63 & $1,06-127,27$ & $27 \%$ & $3 \%$ \\
$\quad$ Neumonía & 5,20 & $1,18-22,89$ & $54 \%$ & $34 \%$ \\
HTA & 4,44 & $1,12-23,15$ & $73 \%$ & $10 \pm 7$ \\
Días UTI & 1,08 & $1,00-1,17$ & $16 \pm 12$ & \\
\hline
\end{tabular}

HTA: Hipertensión arterial. 
resultados previamente reportados en patología cerebrovascular, cuya mortalidad al año de seguimiento varía entre 67 y 91\%2,10,12-14, mientras que para TEC grave, ésta ha sido reportada entre $38 \mathrm{y}$ 45\%1,15-17.

Encontramos que el puntaje APACHE II, el GCS al ingreso y la indicación neurológica de VM representan factores predictores de mortalidad. Varios estudios han identificado éstos y otros factores predictores en pacientes con enfermedad cerebrovascular, entre los que cabe destacar el sexo masculino, la edad, la presencia de patología asociada, como diabetes y patología cardiovascular, AVE de tipo hemorrágico y de ubicación infratentorial 2,12,13,18. El nivel de conciencia es clave en el pronóstico, y son ominosos la pérdida de reflejos de troncoencéfalo y la presencia de Babinski bilateral ${ }^{10,12,13,18,19}$. En estudios de TEC, se ha relacionado un peor pronóstico a un bajo GCS, hipotensión arterial, ausencia de ventilación espontánea, otras lesiones asociadas, mecanismo de la lesión y alteraciones en la tomografía computarizada ${ }^{15,16}$. Por último, factores de manejo respiratorio y de vía aérea se asocian a peor pronóstico, tales como la indicación neurológica de VM e intubación precoz ${ }^{10,12,18}$. La edad no parece ser un factor que haya influido en los buenos resultados de nuestra serie, ya que es similar a la reportada por otros estudios ${ }^{1,10-14}$.

El índice de Barthel se ha ocupado en dos estudios recientes de pacientes con patología cerebrovascular, considerándose un puntaje sobre 60-70 como moderadamente independiente ${ }^{2,10}$. En nuestra serie, el $74 \%$ de los pacientes que sobreviven tuvieron un puntaje $\geq 70$, mientras el $47 \%$ de ellos se recuperan al punto de tener una vida independiente a pesar de su discapacidad (GOS de moderada discapacidad o buena recuperación). Existe variación en los resultados funcionales reportados en patología cerebrovascular. Entre 62 y 69\% tienen un índice de Barthel sobre $60^{2,19}$, mientras otros han reportado $40 \%$ de discapacidad severa ${ }^{12}$, y $46 \%$ de secuelas importantes $^{13}$. En TEC grave (GCS 3-8), el 78\% de los pacientes presentan discapacidad que en el $60 \%$ es severa ${ }^{1}$, y sólo $28 \%$ de los pacientes adultos tiene buena recuperación ${ }^{17}$. Nuestros resultados funcionales también se comparan favorablemente con los reportados en la literatura internacional (Tabla 3).

La decisión de ventilar artificialmente a pacientes neurológicos críticos es especialmente difícil debido a la incertidumbre sobre resultado a largo plazo de cada paciente. Este problema tiene implicancias no sólo médicas, sino también éticas y legales ${ }^{15}$. Lamentablemente existen pocos estudios que evalúen los resultados funcionales a largo plazo, debido a la alta mortalidad y el bajo número de pacientes en las distintas series ${ }^{10,20}$. En nuestro trabajo la edad sobre 70 años, el antecedente de HTA y sobre 6 días en VM son factores que predicen en forma independiente una mala recuperación funcional. Todos estos factores, junto al estado neurológico del paciente deben ser tomados en cuenta al momento de decidir la conexión a VM y de optar por un manejo médico activo.

Limitaciones del estudio. Se realizó seguimiento telefónico y no en policlínico, por otro lado no se realizó doble evaluación de la información obtenida sobre recuperación funcional, lo que puede condicionar cierto sesgo. En segundo lugar nuestro trabajo incluye a un grupo heterogéneo de pacientes con diversas alteraciones fisiopatológicas y patologías asociadas. Esto, sumado a las diversas terapias que recibieron los pacientes, como trombolisis, craneotomías y drenaje de hematomas, dificulta la comparación. Sin embargo, todos ellos requirieron VM invasiva lo que denota la gravedad del compromiso neurológico. Más importante aún, la evolución a largo plazo fue favorable en un importante número de pacientes, incluso en el subgrupo de pacientes con patología cerebrovascular que es el de mayor morbilidad comunicada.

En conclusión, nuestros resultados justifican el manejo activo en UCI por un equipo multidisciplinario en aquellos pacientes neurológicos que requieren $\mathrm{VM}$. 


\section{REFERENCIAS}

1. Thornhill S, Teasdale GM, Murray GD, McEwen J, Roy CW, PenNy KI. Disability in young people and adults one year after head injury: prospective cohort study. BMJ 2000; 320: 1631-5.

2. Santoli F, De Jonghe B, Hayon J, Tran B, Piperaud M, MERRER J ET AL. Mechanical ventilation in patients with acute ischemic stroke: survival and outcome at one year. Intensive Care Med 2001; 27: 1141-6.

3. Bugedo G, Castilo L, Hernández G. Apoyo ventilatorio en pacientes con patología aguda del sistema nervioso central. Rev Méd Chile 1999; 127: 211-21.

4. Teasdale G, Jennett B. Assessment of coma and impaired consciousness. A practical scale. Lancet 1974; 2: 81-4.

5. Knaus WA, Draper EA, Wagner DP, Zimmerman JE. APACHE II: a severity of disease classification system. Crit Care Med 1985; 13: 818-29.

6. Vincent JL, Moreno R, Takala J, Wilatts S, De Mendonca A, Bruining H et al. The SOFA (Sepsis related Organ Failure Assessment) score to describe organ dysfunction/failure. On behalf of the Working Group on Sepsis-Related Problems of the European Society of Intensive Care Medicine. Intensive Care Med 1996; 22: 707-10.

7. Bugedo G, Bruhn A, Segovia V, Zúñiga P, Apablaza F, Bernucci $F$ et al. El Indice de Oxigenación es un mejor indicador del intercambio gaseoso que la relación $\mathrm{PaO}_{2}: \mathrm{FiO}_{2}$ en pacientes adultos sometidos a ventilación mecánica. Rev Chil Medicina Intensiva 2003; 18: 28-33.

8. Jennett B, Bond M. Assessment of outcome after severe brain damage. Lancet 1975; 1: 480-4.

9. Mahoney F, Barthel D. Functional evaluation: The Barthel Index. Maryland State Medical Journal 1965; 14: 56-61.

10. Mayer SA, Copeland D, Bernardini GL, BodenAlbala B, Lennihan L, Kossoff S et al. Cost and outcome of mechanical ventilation for life threatening stroke. Stroke 2000; 31: 2346-53.

11. Diringer MN, EDwards DF, AiYagari V, Howngsworth H. Factors associated with withdrawal of mechanical ventilation in a neurology/neurosurgery intensive care unit. Crit Care Med 2001; 29: 1792-7.

12. Steiner T, Mendoza G, De Georgia M, Schelunger $P$, Howe R, Hacke W. Prognosis of stroke patients requiring mechanical ventilation in a neurological critical care unit. Stroke 1997; 28: 711-5.

13. Burtin P, Bolaert PE, Feldmann L, Nace L, Lelarge P, BaUer P et al. Prognosis of stroke patients undergoing mechanical ventilation. Intensive Care Med 1994; 20: 32-6.

14. Berrouschot J, Rossier A, Koster J, Schneider D. Mechanical ventilation in patients with hemispheric ischemic stroke. Crit Care Med 2000; 28: 2956-61.

15. WAXMAn K, SUndine MJ, Young RF. Is early prediction of outcome in severe head injury possible? Arch Surg 1991; 126: 1237-41; discussion 1242.

16. PaL J, Brown R, FieISZER D. The value of the Glasgow Coma Scale and Injury Severity Score: predicting outcome in multiple trauma patients with head injury. J Trauma 1989; 29: 746-8.

17. Alberico AM, Ward JD, Choi SC, Marmarou A, Young HF. Outcome after severe head injury. Relationship to mass lesions, diffuse injury, and ICP course in pediatric and adult patients. J Neurosurg 1987; 67: 648-56.

18. GujJar AR, Deibert E, Manno EM, Duff S, Diringer MN. Mechanical ventilation for ischemic stroke and intracerebral hemorrhage: indications, timing and outcome. Neurology 1998; 51: 447-51.

19. Bushnell CD, Phimps-Bute BG, Laskowitz DT, Lynch JR, Chilukuri V, Borel CO. Survival and outcome after endotracheal intubation for acute stroke. Neurology 1999; 52: 1374-81.

20. LEKER RR, Ben-Hur T. Prognostic factors in artificially ventilated stroke patients. J Neurol Sci 2000; 176: 83-7. 